\title{
Article \\ A Note on the Periodic Solutions for a Class of Third Order Differential Equations
}

\author{
Zouhair Diab $^{1}$, Juan L. G. Guirao ${ }^{2, * \mathbb{D}}$ and Juan A. Vera ${ }^{3}$ \\ 1 Department of Mathematics and Computer Science, Larbi Tebessi University, 12002 Tebessa, Algeria; \\ zouhair.diab@univ-tebessa.dz \\ 2 Departamento de Matemática Aplicada y Estadística, Universidad Politécnica de Cartagena, \\ Hospital de Marina, 30203 Cartagena, Región de Murcia, Spain \\ 3 Centro Universitario de la Defensa, Academia General del Aire, Universidad Politécnica de Cartagena, \\ 30720 Santiago de la Ribera, Región de Murcia, Spain; juanantonio.vera@upct.es \\ * Correspondence: juan.garcia@upct.es
}

check for

updates

Citation: Diab, Z.; Guirao, J.L.G.; Vera J.A. A Note on the Periodic Solutions for a Class of Third Order Differential Equations. Symmetry 2021, 13, 31. https://dx.doi.org/10.3390/ sym13010031

Received: 9 November 2020 Accepted: 23 December 2020 Published: 27 December 2020

Publisher's Note: MDPI stays neutral with regard to jurisdictional claims in published maps and institutional affiliations.

Copyright: () 2020 by the authors. Licensee MDPI, Basel, Switzerland. This article is an open access article distributed under the terms and conditions of the Creative Commons Attribution (CC BY) license (https: / / creativecommons.org/ licenses/by/4.0/).

\begin{abstract}
The aim of the present work is to study the necessary and sufficient conditions for the existence of periodic solutions for a class of third order differential equations by using the averaging theory. Moreover, we use the symmetry of the Monodromy matrix to study the stability of these solutions.
\end{abstract}

Keywords: differential equations; periodic solution; averaging theory

MSC: 37C80; 37G15; 37C30

\section{Introduction and Statement of the Main Results}

Third-order differential equations

$$
\dddot{x}+g(x, \dot{x}, \ddot{x})=0
$$

are associated with so-called jerk dynamics. In the literature, the third derivative of the position is called the jerk, see [1] and references therein. Many applications of the jerk dynamics to multidisciplinary sciences are been development, see for instance [2].

From a purely mathematical view, various authors have done interesting research about the periodic structure, chaos and other modern topics on nonlinear analysis related to the jerk dynamics; see for example [3-6].

It is well known that the analytical study of the periodic orbits of differential equations is one of the most important problems in qualitative theory. It is in terms of number, existence, non-existence, uniqueness and stability of such periodic orbits. Motivated by it, it will make sense to study the perturbed jerk dynamics, i.e, the third-order differential equations,

$$
\dddot{x}+g(x, \dot{x}, \ddot{x})=\varepsilon f(t, x, \dot{x}, \ddot{x}, \varepsilon),
$$

with $\varepsilon$ is a small parameter and $f, g$ are smooth functions.

In what follows, we endeavor to study the periodic structure of this type of problem.

There are different methods to face such a study. In the literature, there are some papers analyzing the periodic orbits for this class of third order differential equations using the Leray-Schauder method; see [7,8]. There are also some works that use the LeraySchauder method in the field of partial differential equations, for example, studying the existence of weak solutions to the nonlinear elliptic problem [9] and studying the existence of traveling waves for delay reaction-diffusion equations, see [10].

In the present paper, we shall study the periodic orbits of this class of third-order differential equations, by using the averaging theory of dynamical systems, which is 
a completely different approach to the ones used in the mentioned papers. In [11-13] averaging theory was applied to order three systems too.

One of the main problems in the qualitative study of a system of nonlinear differential equations is the investigation of the periodic structure of the problem. Many of the current methods used by researchers who study these problems are born from the ideas and considerations expressed by Henri Poincaré in his treatise [14].

Among the analytical methods considered, the averaging method is a clear candidate to address to the problems of the following type of vectorial differential equations

$$
\frac{d \mathbf{x}}{d t}+f(\mathbf{x})=\varepsilon g(\mathbf{x}, t)
$$

In the literature on this matter, we can go back to Laplace where he introduces the original foundations of the averaging method [15]. Already in the previous century, the averaging theory was theoretically-based. In numerous works, different theoretical and applied results of the development of the theory were collected.

Please note that in modern language, the averaging theory relies on the so-called displacement map of the system and by mean of this method allows obtaining an asymptotic expansion in the small parameter $\varepsilon$ of this map. The zeros of the map which are hyperbolic provide an asymptotic expansion of the initial condition of the periodic orbit. For more details, see [16] and its references.

In [17], Garcia and Llibre studied the periodic structure of second order differential equations of the form

$$
\ddot{x}+g(x)=\varepsilon f(t, x, \dot{x}, \varepsilon),
$$

and they chose a perturbation family of a Duffing equation and forced pendulum in the form:

$$
\ddot{x}+g(x)=\mu^{2 n+1} p(t)+\mu^{4 n+1} q(t, x, \dot{x}, \mu),
$$

where $n \in \mathbb{N}, \mu$ is a small parameter, and

$$
g(x)=x+x^{2 n+1}(b+x h(x))
$$

where $h(x)$ is smooth, $b \neq 0, p(t)$ and $q(t, x, y, \mu)$ are smooth and periodic with period $2 \pi$ in the variable $t$.

Therefore, inspired by this study we decided to study the generalization of this system considering the jerk variable in the form

$$
\dddot{x}+g(x, \dot{x}, \ddot{x})=\mu^{2 n+1} p(t)+\mu^{4 n+1} q(t, x, \dot{x}, \ddot{x}, \mu),
$$

where $n \in \mathbb{N}, \mu$ is a small parameter, and

$$
g(x, \dot{x}, \ddot{x})=-\ddot{x}+\dot{x}-x+(x-\dot{x})^{2 n+1}(b+x h(x)),
$$

and $q(t, x, \dot{x}, \ddot{x}, \mu), p(t)$ are smooth and periodic with period $2 \pi$ in $t, h(x)$ is even smooth function with $b \neq 0$.

We introduce the following variables $(y, z)=(\dot{x}, \ddot{x})$, then we write (2) as the differential system

$$
\begin{aligned}
& \dot{x}=y, \\
& \dot{y}=z \\
& \dot{z}=x-y+z-(x-y)^{2 n+1}(b+x h(x))+\mu^{2 n+1} p(t)+\mu^{4 n+1} q(t, x, y, z, \mu) .
\end{aligned}
$$

Let $\alpha_{1}$ and $\beta_{1}$ be the first Fourier coefficients of $p(t)$, i.e.,

$$
\alpha_{1}=\frac{1}{\pi} \int_{0}^{2 \pi} p(t) \cos t d t, \quad \beta_{1}=\frac{1}{\pi} \int_{0}^{2 \pi} p(t) \sin t d t
$$


and let $\Gamma(x)$ the gamma function.

In the following definition, we are concerned with the limit cycle and its properties:

Definition 1. A periodic orbit in the plane is called a limit cycle if it is an isolated trajectory in the set of all periodic orbits of a differential equation.

The limit cycle is topologically distinguished with neighboring trajectories that are not closed. theorem:

The statement of our main result on the periodic structure of system (2) is the following

Theorem 1. If $\alpha_{1} \beta_{1} b \neq 0, \alpha_{1} \neq \beta_{1}$ then for $\mu \neq 0$ sufficiently small the differential Equation (2) has a periodic solution $x(t, \mu)$ of period $2 \pi$ satisfying the initial condition

$$
x(0, \mu)=\mu \pi^{\frac{1}{4 n+2}}\left(\frac{\Gamma(n+2)}{2 b \Gamma\left(n+\frac{3}{2}\right)}\right)^{\frac{1}{2 n+1}}\left(\alpha_{1}^{2}+\beta_{1}^{2}\right)^{\frac{-n}{1+2 n}} \frac{\left(\alpha_{1}-\beta_{1}\right)}{2}+O\left(\mu^{2 n+1}\right) .
$$

\section{Some Results on the Other Averaging Theory}

We consider the differential system of the form

$$
\dot{\mathbf{x}}=F_{0}(t, \mathbf{x})+\varepsilon F_{1}(t, \mathbf{x})+\varepsilon^{2} R(t, \mathbf{x}, \varepsilon),
$$

with $\varepsilon \neq 0$ is small parameter, $F_{0}, F_{1}: \mathbb{R} \times D \rightarrow \mathbb{R}^{n}$ and $R: \mathbb{R} \times D \times\left(-\varepsilon_{0}, \varepsilon_{0}\right) \rightarrow \mathbb{R}^{n}$ are $\mathcal{C}^{2}$ functions, $2 \pi$-periodic in the first variable, and $D$ is an open subset of $\mathbb{R}^{n}$. We suppose that the differential system

$$
\dot{\mathbf{x}}=F_{0}(t, \mathbf{x}),
$$

has a submanifold of periodic solutions. The system (5) is called the unperturbed system.

In what follows, we denote by $\mathbf{x}(t, \mathbf{z})$ the solution of the unperturbed system (5) satisfying $\mathrm{x}(0, \mathrm{z})=\mathrm{z}$.

We consider the differential system

$$
\dot{\mathbf{y}}=D_{\mathbf{x}} F_{0}(t, \mathbf{x}(t, \mathbf{z})) \mathbf{y},
$$

this system represents the first order variational equation on the periodic solution $\mathbf{x}(t, \mathbf{z})$.

Let $M_{\mathrm{z}}(t)$ be the fundamental matrix of the linear differential system (6) such that $M_{\mathrm{z}}(0)$ be the identity matrix and let the projection $\xi: \mathbb{R}^{k} \times \mathbb{R}^{n-k} \rightarrow \mathbb{R}^{k}$ defined by $\xi\left(x_{1}, \ldots, x_{n}\right)=\left(x_{1}, \ldots, x_{k}\right)$.

In what follows, we denote by $\mathrm{Cl}(\mathrm{V})$ the closure of $V$. The closure of a subset $V$ is the smallest closed subset containing $V$.

Theorem 2. Assume that $V \subset \mathbb{R}^{k}$ be an open and bounded subset and $\beta_{0}: C l(V) \rightarrow \mathbb{R}^{n-k}$ be a $\mathcal{C}^{2}$ function. We consider the following statements:

(i) $\mathcal{Z}=\left\{\mathbf{z}_{\alpha}=\left(\alpha, \beta_{0}(\alpha)\right): \alpha \in C l(V)\right\} \subset D$ and that for every $\mathbf{z}_{\alpha} \in \mathcal{Z}$ the solution $\mathbf{x}\left(t, \mathbf{z}_{\alpha}\right)$ of (5) is periodic of period $T$.

(ii) for every $\mathbf{z}_{\alpha} \in \mathcal{Z}$ there exists a fundamental matrix $M_{\mathbf{z}_{\alpha}}(t)$ of (6) such that the matrix $M_{\mathbf{z}_{\alpha}}^{-1}(0)-M_{\mathbf{z}_{\alpha}}^{-1}(T)$ has in the upper right corner the $k \times(n-k)$ zero matrix, and in the lower right corner $a(n-k) \times(n-k)$ matrix $\Delta_{\alpha}$ with $\operatorname{det}\left(\Delta_{\alpha}\right) \neq 0$.

Let us define the function $\mathcal{F}: \mathrm{Cl}(V) \rightarrow \mathbb{R}^{k}$ as

$$
\mathcal{F}(\alpha)=\xi\left(\int_{0}^{T} M_{\mathbf{z}_{\alpha}}^{-1}(t) F_{1}\left(t, \mathbf{x}\left(t, \mathbf{z}_{\alpha}\right)\right) d t\right) .
$$


If there exists $a \in V$ with $\mathcal{F}(a)=0$ and

$$
\operatorname{det}((d \mathcal{F} / d \alpha)(a)) \neq 0,
$$

then for $\varepsilon \neq 0$ sufficiently small there exists a periodic solution $\mathbf{x}(t, \varepsilon)$ of period $2 \pi$ for differential system (4) such that $\mathbf{x}(0, \varepsilon) \rightarrow \mathbf{z}_{a}$ as $\varepsilon \rightarrow 0$.

For a proof on the existence of the periodic solution of Theorem 2 see Malkin [18] and Roseau [19], or [20] for a proof in a shorter way. By using an idea similar to the one used to proved the stability of the periodic solutions of Theorem 11.6 in [21] we can prove the stability of periodic orbits.

\section{Proof of Theorem 1}

To apply the averaging theory summarized at Section 2 to the differential system (3) we do the following rescaling $x \rightarrow \mu x, y \rightarrow \mu y$ and $z \rightarrow \mu z$. Hence the system (3) writes as follows

$$
\begin{aligned}
\dot{x} & =y, \\
\dot{y} & =z, \\
\dot{z} & =x-y+z+\mu^{2 n}\left(-b(x-y)^{2 n+1}+p(t)\right) \\
& -\mu^{2 n+1} x(x-y)^{2 n+1} h(\mu x)+\mu^{4 n} q(t, \mu x, \mu y, \mu z, \mu),
\end{aligned}
$$

Suppose that $\mu^{2 n}=\varepsilon, O(\varepsilon)=\left(-\mu^{2 n+1} x(x-y)^{2 n+1} h(\mu x)+\mu^{4 n} q(t, \mu x, \mu y, \mu z, \mu)\right)$ and $F(t, x, y, z)=-b(x-y)^{2 n+1}+p(t)$ the system (9) becomes

$$
\begin{aligned}
\dot{x} & =y, \\
\dot{y} & =z, \\
\dot{z} & =x-y+z+\varepsilon\left(-b(x-y)^{2 n+1}+p(t)\right)+O\left(\varepsilon^{2}\right), \\
& =x-y+z+\varepsilon F(t, x, y, z)+O\left(\varepsilon^{2}\right) .
\end{aligned}
$$

The origin is the unique equilibrium point of the unperturbed system $(10)_{\varepsilon=0}$, the eigenvalues of this unperturbed system at the origin are $i,-i, 1$. We do the following linear change

$$
(X, Y, Z)^{T}=C(x, y, z)^{T},
$$

where the matrix

$$
C=\left(\begin{array}{ccc}
1 & -1 & 0 \\
0 & -1 & 1 \\
1 & 0 & 1
\end{array}\right)
$$

System (10) is transformed into the next system by using the previous linear change

$$
\begin{aligned}
& X^{\prime}=-Y, \\
& Y^{\prime}=X+\varepsilon \tilde{F}(t, X, Y, Z)+O\left(\varepsilon^{2}\right), \\
& Z^{\prime}=Z+\varepsilon \tilde{F}(t, X, Y, Z)+O\left(\varepsilon^{2}\right),
\end{aligned}
$$

where the linear part of this system in the real Jordan canonical normal form and

$$
\tilde{F}(t, X, Y, Z)=F\left(t, \frac{X-Y+Z}{2}, \frac{-X-Y+Z}{2}, \frac{-X+Y+Z}{2}\right) .
$$

Returning to (4), we consider $\mathbf{x}=(X, Y, Z), F_{0}(t, \mathbf{x})=(-Y, X, Z), F_{1}(t, \mathbf{x})=(0, \tilde{F}, \tilde{F})$ and $F_{2}(t, \mathbf{x})=\left(0, O\left(\varepsilon^{2}\right), O\left(\varepsilon^{2}\right)\right)$. Let $\mathbf{x}\left(t, X_{0}, Y_{0}, Z_{0}, \varepsilon\right)$ be the solution to system (11) with $\mathbf{x}\left(0, X_{0}, Y_{0}, Z_{0}, \varepsilon\right)=\left(X_{0}, Y_{0}, Z_{0}\right)$. Please note that the unperturbed system $(11)_{\varepsilon=0}$ has a 
linear center at the origin in the $(X, Y)$ plane, and the periodic solution $\mathbf{x}\left(t, X_{0}, Y_{0}, 0,0\right)=$ $(X(t), Y(t), Z(t))$ is

$$
X(t)=X_{0} \cos t-Y_{0} \sin t, \quad Y(t)=Y_{0} \cos t+X_{0} \sin t, \quad Z(t)=0 .
$$

Please note that $(X(t), Y(t), Z(t))$ has period $2 \pi$. For our system, $V$ and $\alpha$ from Theorem 2 are

$$
V=\left\{(X, Y, 0): 0<X^{2}+Y^{2}<\rho\right\},
$$

where $\rho>0$ is arbitrary and $\alpha=\left(X_{0}, Y_{0}\right) \in V$.

The fundamental matrix $M_{\mathbf{z}}(t)=M(t)$ of the first order variational equation (6) satisfying the system $(11)_{\varepsilon=0}$ is

$$
M(t)=\left(\begin{array}{ccc}
\cos t & -\sin t & 0 \\
\sin t & \cos t & 0 \\
0 & 0 & e^{t}
\end{array}\right),
$$

We note that $M(t)$ is independent from the initial condition $\left(X_{0}, Y_{0}, 0\right)$, and

$$
M^{-1}(0)-M^{-1}(2 \pi)=\left(\begin{array}{ccc}
0 & 0 & 0 \\
0 & 0 & 0 \\
0 & 0 & 1-e^{-2 \pi}
\end{array}\right) .
$$

Very briefly, we confirmed that the statements of Theorem 2 hold. Now we study the solutions $\alpha=\left(X_{0}, Y_{0}\right) \in V$ of the function $\mathcal{F}(\alpha)$ given in (7). In other words, we have $\mathcal{F}(\alpha)=\left(\mathcal{F}_{1}(\alpha), \mathcal{F}_{2}(\alpha)\right)$ where

$$
\begin{gathered}
\mathcal{F}(\boldsymbol{\alpha})=\int_{0}^{2 \pi} M^{-1}(t) F_{1}\left(t, \mathbf{x}\left(t, \mathbf{z}_{\alpha}, 0\right)\right) d t=\left(\begin{array}{c}
\mathcal{F}_{1}\left(X_{0}, Y_{0}\right) \\
\mathcal{F}_{2}\left(X_{0}, Y_{0}\right)
\end{array}\right)= \\
\left(\begin{array}{c}
\int_{0}^{2 \pi} \sin (t) \tilde{F}\left(t, \mathbf{x}\left(t, X_{0}, Y_{0}, 0,0\right)\right) d t \\
\int_{0}^{2 \pi} \cos (t) \tilde{F}\left(t, \mathbf{x}\left(t, X_{0}, Y_{0}, 0,0\right)\right) d t
\end{array}\right)= \\
\left(\begin{array}{c}
\int_{0}^{2 \pi} \sin (t) F\left(t, \frac{X(t)-Y(t)}{2},-\frac{X(t)+Y(t)}{2}, \frac{-X(t)+Y(t)}{2}\right) d t \\
\int_{0}^{2 \pi} \cos (t) F\left(t, \frac{X(t)-Y(t)}{2},-\frac{X(t)+Y(t)}{2}, \frac{-X(t)+Y(t)}{2}\right) d t
\end{array}\right)= \\
\left(\begin{array}{c}
\int_{0}^{2 \pi} \sin (t)\left(-b\left(X_{0} \cos t-Y_{0} \sin t\right)^{2 n+1}+p(t)\right) d t \\
\int_{0}^{2 \pi} \cos (t)\left(-b\left(X_{0} \cos t-Y_{0} \sin t\right)^{2 n+1}+p(t)\right) d t
\end{array}\right),
\end{gathered}
$$

Using simple calculations, it can be shown that

$$
\begin{aligned}
& \int_{0}^{2 \pi} \sin t\left(X_{0} \cos t-Y_{0} \sin t\right)^{2 n+1} d t=-\frac{2 \sqrt{\pi} \Gamma\left(\frac{3}{2}+n\right)}{\Gamma(2+n)} Y_{0}\left(X_{0}^{2}+Y_{0}^{2}\right)^{n}, \\
& \int_{0}^{2 \pi} \cos t\left(X_{0} \cos t-Y_{0} \sin t\right)^{2 n+1} d t=\frac{2 \sqrt{\pi} \Gamma\left(\frac{3}{2}+n\right)}{\Gamma(2+n)} X_{0}\left(X_{0}^{2}+Y_{0}^{2}\right)^{n} .
\end{aligned}
$$

We solve the following system

$$
\left(\begin{array}{c}
\mathcal{F}_{1}\left(X_{0}, Y_{0}\right) \\
\mathcal{F}_{2}\left(X_{0}, Y_{0}\right)
\end{array}\right)=\left(\begin{array}{c}
b \frac{2 \sqrt{\pi} \Gamma\left(\frac{3}{2}+n\right)}{\Gamma(2+n)} Y_{0}\left(X_{0}^{2}+Y_{0}^{2}\right)^{n}+\pi \beta_{1} \\
-b \frac{2 \sqrt{\pi} \Gamma\left(\frac{3}{2}+n\right)}{\Gamma(2+n)} X_{0}\left(X_{0}^{2}+Y_{0}^{2}\right)^{n}+\pi \alpha_{1}
\end{array}\right)=\left(\begin{array}{l}
0 \\
0
\end{array}\right) .
$$


We obtain a unique solution

$$
\left(\begin{array}{c}
X_{0}^{*} \\
Y_{0}^{*}
\end{array}\right)=\pi^{\frac{1}{4 n+2}}\left(\frac{\Gamma(n+2)}{2 b \Gamma\left(n+\frac{3}{2}\right)}\right)^{\frac{1}{2 n+1}}\left(\alpha_{1}^{2}+\beta_{1}^{2}\right)^{\frac{-n}{1+2 n}}\left(\begin{array}{c}
\alpha_{1} \\
\beta_{1}
\end{array}\right) .
$$

Returning to (8), the $\operatorname{det}\left(D \mathcal{F}\left(X_{0}^{*}, Y_{0}^{*}\right)\right)$ is

$$
(1+2 n) \pi^{\frac{4 n+1}{2 n+1}}\left(\frac{\Gamma(n+2)}{2 b \Gamma\left(n+\frac{3}{2}\right)}\right)^{-\frac{2}{1+2 n}}\left(\alpha_{1}^{2}+\beta_{1}^{2}\right)^{\frac{2 n}{1+2 n}},
$$

in order because $\alpha_{1} \beta_{1} b \neq 0$, the statements of Theorem 2 hold. Using the following relationship

$$
x(t)=\frac{X(t)-Y(t)+Z(t)}{2}
$$

where $X(t), Y(t), Z(t))$ are given by (12), we can prove that

$$
\begin{aligned}
x(0, \mu) & =\mu \frac{X_{0}^{*}-Y_{0}^{*}}{2}+O\left(\mu^{2 n+1}\right) \\
& =\mu \pi^{\frac{1}{4 n+2}}\left(\frac{\Gamma(n+2)}{2 b \Gamma\left(n+\frac{3}{2}\right)}\right)^{\frac{1}{2 n+1}}\left(\alpha_{1}^{2}+\beta_{1}^{2}\right)^{\frac{-n}{1+2 n}} \frac{\left(\alpha_{1}-\beta_{1}\right)}{2}+O\left(\mu^{2 n+1}\right) .
\end{aligned}
$$

This completes the proof of Theorem 1.

Remark 1. The eigenvalues of the Jacobian matrix $D \mathcal{F}\left(X_{0}^{*}, Y_{0}^{*}\right)$ are

$$
\lambda_{1,2}= \pm i(2 n+1)^{\frac{1}{2}} \pi^{\frac{4 n+1}{4 n+2}}\left(\frac{2 b \Gamma\left(\frac{3}{2}+n\right)}{\Gamma(2+n)}\right)^{\frac{4 n^{2}+2 n+1}{2 n+1}}\left(\alpha_{1}^{2}+\beta_{1}^{2}\right)^{\frac{n}{1+2 n}} .
$$

In consequence, we cannot use the averaging theory found in Section 2 to study the stability of the periodic solution of the differential system (10). To solve this problem we use the symmetry of the Monodromy matrix.

The variational equation of this periodic orbit is a given by

$$
\dot{\mathbf{y}}=A(t) \mathbf{y},
$$

where $\mathbf{y}=(x, y, z) \in \mathbb{R}^{3}$ and $A(t)$ a $2 \pi$-periodic matrix given by

$$
A(t)=A_{0}+\varepsilon A_{1}(t) .
$$

with

$$
\begin{aligned}
A_{0} & =\left(\begin{array}{ccc}
0 & 1 & 0 \\
0 & 0 & 1 \\
1 & -1 & 1
\end{array}\right), \\
A_{1}(t) & =\left(\begin{array}{ccc}
0 & 0 & 0 \\
0 & 0 & 0 \\
-b(2 n+1)(x(t)-y(t))^{2 n} & b(2 n+1)(x(t)-y(t))^{2 n} & 0
\end{array}\right)
\end{aligned}
$$

The multipliers of the monodromy matrix of the variational equation are $1+O\left(\varepsilon^{2}\right), 1+O\left(\varepsilon^{2}\right)$ and $e^{2 \pi}+O\left(\varepsilon^{2}\right)$. From it, we conclude that the periodic solution of the differential system (10) is unstable because the multiplier $e^{2 \pi}+O\left(\varepsilon^{2}\right)>1$. 


\section{Conclusions}

Appropriately introducing the small parameter enables us to apply the averaging theory to prove analytically the existence of periodic solutions for a class of third-order differential equations. The application of this theory to the third-order differential equations gave interesting results on the stability of the periodic orbits. Our future work will consist of applying this theory to differential equations of order $n$, with $n \geq 4$ with particular physical applications.

Author Contributions: Investigation, Z.D., J.L.G.G. and J.A.V. All authors have read and agreed to the published version of the manuscript.

Funding: Foundation Seneca: 20783/PI/18 Ministry of Science, Innovation and Universities: PGC2018097198-B-I00.

Data Availability Statement: This paper has not data involved.

Conflicts of Interest: The authors declare no conflict of interest.

\section{References}

1. Schot, S.H. Jerk: The time rate of change of acceleration. Am. J. Phys. 1978, 46, 1090. [CrossRef]

2. Zeithamer, T. Methodology of Theoretical Physics in Economics: Jerk Dynamics. Procedia Econ. Financ. 2015, 30, 967-973. [CrossRef]

3. Gottlieb, H.P.W. Simple nonlinear jerk functions with periodic solutions. Am. Phys. 1998, 66, 903-906. [CrossRef]

4. Patidar, V.; Sud, K.K. Bifurcation and chaos in simple jerk dynamical systems. Pramana 2005, 64, 75-93. [CrossRef]

5. Sprott, J.C. Some simple chaotic jerk functions. Am. J. Phys. 1997, 65, 537-543. [CrossRef]

6. Spprott, J.C. Elegant Chaos. Algebraically Simple Chaotic Flows; World Scientific: Singapore, 2010.

7. Reissig, R. Periodic solutions of a third order nonlinear differential equation. Ann. Mat. Pura Appl. 1972, 92, 193-198. [CrossRef]

8. Xiankai, H. On the existence of periodic solutions for the third-order nonlinear ordinary differential equations. Appl. Math.-A J. Chin. Univ. 1999, 34, 125-130. [CrossRef]

9. Jung, T.; Choi, Q.H. Existence of four solutions for the elliptic problem with nonlinearity crossing one eigenvalue. J. Inequal. Appl. 2013, 2013, 187. [CrossRef]

10. Volpert, V. Existence of waves for a bistable reaction-diffusion system with delay. J. Dyn. Differ. Equ. 2020, 32, 615-629. [CrossRef]

11. Llibre, J.; Roberto, L. On the periodic orbits of the third-order differential equation $x^{\prime \prime \prime}-\mu x^{\prime \prime}+x^{\prime}-\mu x=\varepsilon F\left(x, x^{\prime}, x^{\prime \prime}\right)$. Appl. Math. Lett. 2014, 26, 425-430. [CrossRef]

12. Llibre, J.; Yu, J.; Zhang, X. Limit Cycles for a Class of Third-Order Differential Equations. Rocky Mount. J. Math. 2010, 40, 581-594. [CrossRef]

13. Toniol Cardin, P.; Llibre, J. Transcritical and zero-Hopf bifurcations in the Genesio system. Nonlinear Dyn. 2017, 88, 547-553. [CrossRef]

14. Poincaré, H. Les méthodes Nouvelles de la Mécanique Céleste; Gauthier-Villars: Paris, France, 1989; Volume 3.

15. Marquis de Laplace, P.S. Traité de Mécanique Céleste; Bachelier: 1829; Volume 1.

16. Llibre, J.; Novaes, D.D.; Teixeira, M.A. Higher order averaging theory for finding periodic solutions via Brouwer degree. Nonlinearity 2014, 27, 563-583. [CrossRef]

17. Garcia, A.; Llibre, J. Existence of Periodic Solutions for a Class of Second Order Ordinary Differential Equations. Acta Appl. Math. Vol. 2020, 169, 193-197. [CrossRef]

18. Malkin, I.G. Some Problems of the Theory of Nonlinear Oscillations; State Pub. House of Technical and Theoretical Literature Moscow: Moscow, Russia, 1956. (In Russian)

19. Roseau, M. Vibrations Non Linéaires et Théorie de la Stabilité; Springer Tracts in Natural Philosophy; Springer: New York, NY, USA, 1976; Volume 8.

20. Buică, A.; Françoise, J.P.; Llibre, J. Periodic solutions of nonlinear periodic differential systems with a small parameter. Commun. Pure Appl. Anal. 2006, 6, 103-111. [CrossRef]

21. Verhulst, F. Nonlinear Differential Equations and Dynamical Systems, Universitext; Springer: Berlin, Germany, 1996. 